\title{
Neurophysiologische Befunde bei den Stiff Man-Syndromen
}

\author{
Neurophysiological Findings in the Stiff Man Syndromes
}

\author{
Autor \\ H.-M. Meinck \\ Institut \\ Neurologische Universitätsklinik Heidelberg
}

\section{Schlüsselwörter \\ - Stiff Man-Syndrome \\ - Stiff Person-Syndrome \\ - PERM \\ - Myoklonus \\ antineuronale Autoimmunität}

Key words

- stiff man syndromes

- stiff person syndromes

- PERM

myoclonus

- antineuronal autoimmunity

\section{Bibliografie}

DOI http://dx.doi.org/

10.1055/s-0034-1395513

Klin Neurophysiol 2014;

45: 207-213

(c) Georg Thieme Verlag KG

Stuttgart · New York

ISSN 1434-0275

Korrespondenzadresse

Prof. Dr. Hans-Michael Meinck

Neurologische Universitäts-

klinik

Im Neuenheimer Feld 400

69120 Heidelberg

hans-michael.meinck@med.

uni-heidelberg.de

\section{Zusammenfassung}

$\nabla$

In einem sich klinisch und immunologisch zunehmend ausdifferenzierenden Manifestationsspektrum chronischer Autoimmun- Enzephalomyelitiden sind fluktuierende Rigidität und Spasmen das ungewöhnliche gemeinsame klinische Merkmal der Stiff Man-Syndrome (SMS). Die Autoimmunität richtet sich beim SMS vor allem gegen Proteine inhibitorischer ZNS-Synapsen. Korrespondierend lassen sich neurophysiologische Zeichen einer defekten Inhibition auf verschiedenen motorischen Regulationsebenen nachweisen, besonders im Rückenmark und Hirnstamm, aber auch im motorischen Kortex. Diagnostisch wertvoll ist vor allem die EMGPolygrafie spontaner und reizinduzierter Spasmen und Myoklonien, weil sie reproduzierbare abnorme Innervationsmuster mit kurzer Latenz identifiziert.

\section{Das Syndrom, seine Varianten und Pathogenese \\ $\nabla$}

Syndrom-Definition

Als ,Stiff man-Syndrom' bezeichneten Moersch and Woltman [1] ironisierend die ungewöhnliche und rätselhafte Kombination von fluktuierender Rigidität der Rumpf- und rumpfnahen Muskulatur mit überlagernden schmerzhaften Spasmen ohne weitere Zeichen einer neurologischen Erkrankung. Diese diagnostischen Kernkriterien waren bei den von ihnen beschriebenen Patienten variabel assoziiert mit weiteren schwer zuzuordnenden Auffälligkeiten: ängstliche Gangstörung mit Blockaden und Stürzen, Hyperlordose, episodische adrenerge Dysregulation, Schreckhaftigkeit und ausgeprägte Angst, auf offenen Flächen frei zu gehen oder zu stehen. Entsprechend häufig war die Fehldiagnose einer psychogenen Bewegungsstörung - sie ist es bis heute geblieben ( $\bullet$ Infobox 1$)$ !

\section{Abstract \\ $\nabla$}

In a broadening clinical and immunological spectrum of chronic autoimmune encephalomyelitis, fluctuating rigidity and spasms, an unusual combination, are the common characteristics of stiff man syndromes. Autoimmunity is targeted mainly against proteins of inhibitory CNS synapses. Correspondingly, neurophysiological techniques demonstrate a lack of inhibition at various levels of motor regulation, mainly in the spinal cord and brainstem, but also in the motor cortex. EMG polymyography, particularly of spontaneous and stimulus-induced spasms and jerks, helps one to make the diagnosis by identifying reproducible abnormal innervation patterns with a short latency.

\section{Infobox 1}

Kernsymptome und weitere häufige

Merkmale des SMS und seiner Varianten in der Heidelberger Kohorte $(\mathrm{N}=145)$

Rigidität der axialen Muskulatur $\quad 100 \%$

Schmerzhafte Spasmen $\quad 100 \%$

Gangstörung, -blockaden, Stürze $\quad 85 \%$

Agoraphobie (aufgaben-spezifisch) 80\%

Skelettdeformitäten (Ankylosen) 75\%

Fehldiagnose psychogene Störung $\quad 73 \%$

gesteigerte Schreckhaftigkeit $\quad 70 \%$

gesteigerte Eigenreflexe $\quad 67 \%$

krisenhafte Dysautonomie $\quad 58 \%$

Kopfretraktionsreflex $57 \%$

„Plus“-Symptome (= PERM)* 38\%

*z. B. Okulomotorikstörungen, Ataxie, Paresen, Dysarthrie/Dysphagie, Babinski-Zeichen, Epilepsie 


\section{Syndrom-Varianten}

Heute wird deutlich, dass der ikonische Name Stiff-Man Syndrom (SMS) einen ungewöhnlichen Subtyp von chronischen Autoimmun-Enzephalomyelitiden bezeichnet, bei dem Rigidität und Spasmen das verbindende Merkmal innerhalb eines sich klinisch und immunologisch zunehmend ausdifferenzierenden Manifestationsspektrums bilden. Fälle mit axialer Rigidität und Spasmen mit weiteren neurologischen Symptomen wie z. B. Ataxie, Augenbewegungsstörung, fokale Epilepsie, Pyramidenbahnzeichen, zentraler oder peripherer Lähmung werden als PlusVariante angesehen und - in Distinktion zum klassischen SMS von den meisten Autoren als progressive Enzephalomyelitis mit Rigidität und Myoklonien (PERM) bezeichnet. Rigidität und Spasmen nur einer Gliedmaße, meist eines Beines, Stiff limb-Syndrom oder Stiff leg-Syndrom (SLS), gelten als MinusVariante des klassischen SMS [2-5]. Klinische und immunologische Charakteristika dieser Krankheitsbilder sind kürzlich an anderer Stelle dargestellt worden [6]. Der Schwerpunkt dieses Beitrages liegt, der thematischen Ausrichtung der Zeitschrift folgend, auf den klinisch-neurophysiologischen Aspekten.

\section{Pathogenese}

Als Ursache vermutete man zunächst eine neuromuskuläre Störung. Pathologisch-anatomische Hinweise auf eine entzündliche Erkrankung des ZNS wurden anderen Krankheitsentitäten zugeordnet (,spinale Neuronitis', [7,8], Übersicht bei [4]). Charakteristische neurophysiologische Normabweichungen und ihre Modulation durch zentral wirksame Pharmaka öffneten den Blick dafür, dass es sich um eine zentrale Bewegungsstörung handelt Übersicht in [9].

Verschiedene Befunde sprechen für eine Autoimmun-Pathogenese ( $\bullet$ Infobox 2). Die dominante Autoimmunität richtet sich gegen Glutamat-Dekarboxylase (GAD, etwa $80 \%$ in der Heidelberger Kohorte). GAD reguliert die Synthese des ZNS-Transmitters GABA. Hieraus wurde die Hypothese abgeleitet, dass die Bewegungsstörung verursacht wird durch eine Autoimmunattacke gegen hemmende GABAerge Neurone, mithin als motorische Disinhibitions-Symptomatik zu verstehen ist. Autoimmunität gegen weitere Proteine hemmender Synapsen, z.B. Glyzinrezeptor Typ 1 (GlyR1; 12\%), Amphiphysin 1 (ca. 6\%) und andere neuronale Antigene (etwa 4\%) lassen sich mit dem Kerngedanken dieser Hypothese gut vereinbaren [13-15]. Allerdings kommen Serum-Ak gegen GAD im Kontext zahlreicher - auch nicht-neurologischer Erkrankungen und gelegentlich sogar bei Gesunden vor [14].

\section{Infobox 2}

Argumente für die Autoimmun-Pathogenese der

Stiff Man-Syndrome

- hohe Prävalenz für Autoimmun-Endokrinopathien (etwa 70\%, z.B. Diabetes mellitus Typ I, Hypo- und Hyperthyreose, atrophische Gastritis, B12-Hypovitaminose) mit charakteristischen organspezifischen Autoantikörpern (Ak) [10];

- hohe Prävalenz von Ak gegen neuronale Proteine in Serum und Liquor (GAD, Amphiphysin 1, Glyzin-Rezeptoren, DPPX, $\mathrm{GABA}_{\mathrm{A}}$-Rezeptoren; insgesamt 80-90\%);

- intrathekale de novo Synthese von antineuronalen Ak, IgG oder oligoklonalen Banden;

- Übertragung der Symptome auf Labortiere mit IgG von Patienten [11,12];

- Wirksamkeit immunmodulierender Therapien [Übersicht bei 13].

\section{Klinische Manifestation}

$\nabla$

\section{Epidemiologie und Verlauf}

Das mittlere Manifestationsalter liegt in der Heidelberger Kohorte $(\mathrm{N}=145)$ bei $46(18-81)$ Jahren; $2 / 3$ der Patienten sind weiblich. Der typische Verlauf ist charakterisiert durch schleichenden Beginn und langsame Progression über Monate oder Jahre mit Übergang in ein stabiles Stadium. Neben dieser typischen kommt deutlich seltener eine Verlaufsform mit subakuten Schüben vor, vor allem bei der PERM-Variante mit Antikörpern gegen Glyzin-Rezeptoren [15]. Spontane Remissionen sind sehr selten $[4,6]$.

\section{Kernsymptome}

Die häufigeren klinischen Symptome zeigt $\bullet$ Infobox 1; die ausführliche Darstellung beschränkt sich auf die obligaten Kernsymptome. Die Rigidität ist meist in den Muskeln der unteren Rumpfhälfte lokalisiert und symmetrisch, sie fluktuiert zwischen kaum merklich (z.B. in Ruhe, im Schlaf oder in Narkose) und intensiv (bei Beobachtung oder Provokation). Spontane Spasmen zeigen meist ein rasches An- und langsames Abklingen, typischerweise mit opisthotonischem Streckmuster des Rumpfes. Oft beginnen die Spasmen jedoch auch ruckartig (myoklonisch) und so kraftvoll (und schmerzhaft), dass sie Muskelrisse oder sogar „Spontan“-Frakturen verursachen können. Typischerweise besteht eine deutlich gesteigerte Reaktivität der Patienten bei exterozeptiver Stimulation (Berührung, Schmerz, Desinfektionsspray). Auch banale akustische Signale (Telefonklingel, Türöffnung) oder unerwartete Berührungen lösen gesteigerte Schreckreaktionen aus (Startle, Hyperekplexie). Etwa $60 \%$ der Patienten haben einen brüsken Kopfretraktionsreflex bei Beklopfen der Stirn oder des Nasenrückens, gelegentlich auch andere atavistische Reflexe des Gesichts zusätzlich zu den gesteigerten Muskeleigenreflexen [16].

Rigidität und Spasmen verursachen Dystonie-ähnliche Ankylosen oder Subluxationen, am häufigsten eine fixierte Hyperlordose, die sich beim Vorbeugen nicht ausgleicht. Auch neurogene Fußdeformitäten (z. B. Hohl-Spitzfuß, Hakenzehen) oder ein als Hallux rigidus fehldiagnostizierter, striatal toe sind nicht selten. Das Gangmuster wirkt ängstlich, steifbeinig und oft bizarr. Zunehmende Anforderungen (z.B. Gehen in der Mitte des Flures oder im Freien, treppab ohne Berührung des Handlaufs) verursachen Gangblockaden, oft assoziiert mit Myoklonien oder Spasmen mit konsekutiven unkontrollierten Stürzen und erheblichem Verletzungsrisiko.

\section{Hilfsuntersuchungen}

Ak gegen neuronale Antigene, findet man bei $80-90 \%$ der Patienten. Ak gegen mehrere neuronale Antigene sind keineswegs selten. Nicht zuletzt deshalb sollten immer sämtliche infrage kommenden antineuronalen Ak untersucht werden (Infobox 2). Einerseits eröffnet der Nachweis einer Autoimmunität gegen neuronale Oberflächenproteine (z.B. GlyR1 oder DPPX) andere therapeutische Optionen als der Nachweis einer Autoimmunität gegen zytosolische neuronale Antigene (GAD, Amphiphysin 1) [14]. Andererseits weisen insbesondere die Ak gegen Amphiphysin 1, seltener die gegen GlyR1 auf ein okkultes Neoplasma hin. Im Liquor lassen sich die antineuronalen Ak mit erhöhten AkIndizes nachweisen, daneben besteht bei etwa $60 \%$ eine intrathekale de novo Synthese von IgG und/oder oligoklonale Banden, in etwa $20 \%$ auch eine milde Lymphozytose. Kernspintomogram- 
me von Gehirn und Rückenmark zeigen dagegen keine spezifischen Befunde [6].

\section{Therapie}

Rigidität, Spasmen und aufgaben-spezifische Phobie lassen sich am effektivsten mit Benzodiazepinen unterdrücken. Baclofen und Tizanidine, gelegentlich auch Valproat oder Pregabalin helfen als add-on Medikation, die Benzodiazepin-Dosis niedrig zu halten [9]. Nachlassende Wirkung ist gelegentlich auf ein Fortschreiten der Erkrankung zurückzuführen, häufiger auf eine Benzodiazepin-Adaptation - dann sollte man sich nicht scheuen, die Dosierung dem Bedarf anzupassen. Immunmodulierende Strategien, in erster Linie Immunglobuline und Methylprednisolon, wirken meist erst nach wochen- bis monatelanger Behandlung. Bei wiederholter Anwendung kann die Wirksamkeit ebenfalls nachlassen. Patienten mit Ak gegen Proteine der neuronalen Zell-Oberfläche scheinen insgesamt häufiger und stärker von einer immunmodulierenden Therapie zu profitieren als Patienten mit Ak gegen zytosolische Antigene [13,14].

\section{Klinische Neurophysiologie \\ $\nabla$}

\section{Standarduntersuchungen}

Als charakteristisch für das SMS gilt im EMG das kontinuierliche Feuern normaler motorischer Einheiten mit niedriger, auffallend stabiler Frequenz (Continuous Motor Unit Activity, CMUA) vor allem in der lumbalen Paraspinalmuskulatur ( $\bullet$ Abb. 1). Diese Aktivität können die Patienten willentlich kaum oder gar nicht unterdrücken. Man sieht CMUA in ähnlicher Weise auch bei anderen Erkrankungen mit erhöhtem muskulären Ruhetonus, z. B. beim Parkinsonismus, bei der kongestiven Myelopathie oder beim schmerzbedingten Hartspann. Es handelt sich hierbei also sicher nicht um einen spezifischen diagnostischen Befund, sondern allenfalls um ein Warnsignal. Allerdings verschwindet CMUA beim SMS im Schlaf, bei paralytischer Regionalanästhesie und in Narkose - was immerhin belegt, dass sie im ZNS generiert wird. Neben der CMUA am häufigsten abnorm ist der Masseter-Hemmungsreflex, der bei etwa $30 \%$ der Patienten einen Verlust seiner S2-Komponente zeigt ( $\bullet$ Abb. 2). Dieser Befund deutet auf eine mangelhafte Ausprägung neuronaler Hemmungsmechanismen im Hirnstamm hin und wird in ähnlicher Form auch bei Patienten mit Hyperekplexie beobachtet, einer familiären oder sporadischen Störung der glyzinergen Inhibition [17]. Die anderen elektrophysiologischen Standarduntersuchungen (EEG, NLG, EP, TKMS) zeigen häufig keine richtungsweisenden

\section{Infobox 3}

Differenzialdiagnose der Stiff Man-Syndrome

A Rheumatische und neuromuskuläre Erkrankungen

Mb. Bechterew

Myositis fibrosa generalisata

Rigid spine-Syndrom

Myophosphorylase-Mangel (McArdle-Erkrankung)

Atypische Polyradikulitis

Neuromyotonie (Isaacs-/Morvan-Syndrom)

Cramping disease (Satoyoshi-Syndrom)

\section{B ZNS-Erkrankungen}

Tetanus

Strychnin Intoxikation

Hyperekplexie

Intramedulläre spinale Tumore

Atypische Myelitis

Axiale Dystonie

Orthostatischer Tremor

Psychogene Bewegungsstörung

Normabweichungen sondern allenfalls Auffälligkeiten, die offensichtlich im Zusammenhang stehen mit einer begleitenden diabetischen Polyneuropathie. Immerhin klärt aber die klinisch-neurophysiologische Routinediagnostik einen Großteil der infrage kommenden Differenzialdiagnosen ( 0 Infobox 3 ). Bei der kortikalen Magnetstimulation sieht man gelegentlich eine abnorme Komponente, welche die kortikale Silent Period durchbricht [18] und vermutlich einem Reflex-Myoklonus entspricht. Außerhalb der routinediagnostischen Fragestellung lässt sich mit dieser Methode eine reduzierte intrinsische Inhibition im motorischen Kortex nachweisen, die mit der Antikörperkonzentration im Liquor korreliert [19]. Ob dies zur Erhöhung des Muskeltonus und den Spasmen beiträgt, ist unklar. Mit speziellen reflexphysiologischen Methoden lassen sich komplexe Störungen spinaler Hemmungsmechanismen nachweisen [20].

\section{EMG-Polygrafie von Spasmen und Myoklonien}

Die EMG-Polygrafie ermöglicht mit exzellentem zeitlichem Auflösungsvermögen die Darstellung und Analyse von Spasmen und Myoklonien, die wegen ihrer Geschwindigkeit, Flüchtigkeit oder Polytopie unter rein klinischen Bedingungen kaum möglich sind. Messung von Latenzen, Identifizierung von abnormen Innervationsmustern und Feststellung ihrer Reproduzierbarkeit erlauben diagnostische Rückschlüsse. So kann in den meisten

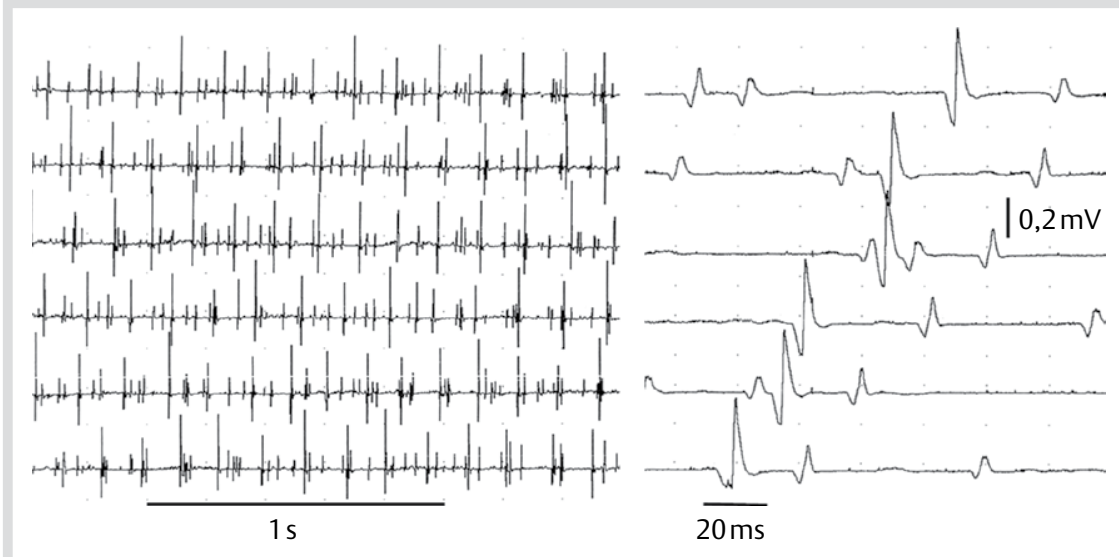

Abb. 1 Continuous Motor Unit Activity (CMUA) Fortlaufende Registrierung mit Nadelelektrode, langsame (links) und schnelle (rechts) Registriergeschwindigkeit. Beachte die niedrige, aber stabile Entladungsfrequenz der normal geformten MUAPs, sodass bei hoher Registriergeschwindigkeit gelegentlich ein ,stehendes Bild` entsteht. 
Fällen die Differenzialdiagnose einer psychogenen Bewegungsstörung definitiv verworfen oder eben auch wahrscheinlich gemacht werden [21]. Allerdings bedarf es zur Durchführung solcher komplexer Untersuchungen eines gewissen Trainings und der Möglichkeit, von mehreren Muskeln simultan abzuleiten. Bereits die einkanalige EMG-Ableitung spontaner Spasmen zeigt, dass die Muskelaktivität hierbei ungewöhnlich gleichmäßig zu- oder abnehmen kann ( $\bullet$ Abb. 3a, b). Die MehrkanalAbleitung belegt, dass die Innervation verschiedener Muskeln bei den myoklonischen Spasmen mit abnormen Mustern erfolgt, welche sich durch eine ausgeprägte Hypersynchronie auszeichnen: bi- oder triphasische EMG-Bursts mit hohen Amplituden, die in benachbarten Muskeln, insbesondere in Antagonistenpaaren, simultan auftreten und die häufig von einer ebenfalls syn-

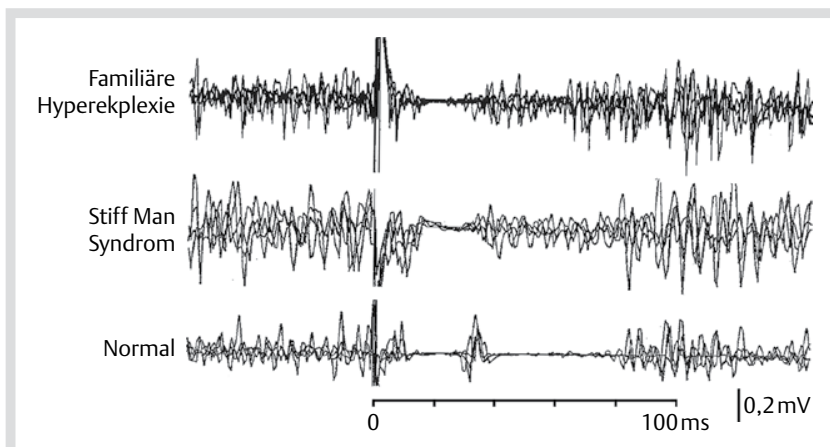

Abb. 2 Masseter-Hemmungsreflexe ausgelöst durch Elektrostimulation der Unterlippe mit hoher Reizstärke (Rechteckimpuls, > 3 x Reflexschwelle) bei jeweils einem Patienten mit familiärer Hyperekplexie, SMS und einem Gesunden. Jeweils 4 Reflexe superponiert. Reiz bei $0 \mathrm{~ms}$. Beim Gesunden wird mit Impulsen > 3 x Reflexschwelle die Vorinnervation zwischen etwa 10 und 100 ms biphasisch blockiert, während sie bei SMS und Hyperekplexie selbst mit weiter ansteigender Reizstärke nur unvollständig unterdrückt wird. chronen Innervationspause gefolgt sind ( $\bullet$ Abb. 3c). Derartige Aktivitätsformen sind ausnahmslos pathologisch, nämlich myoklonisch. Insofern erlaubt ihre Registrierung auf Anhieb die klinisch nicht immer einfache Differenzierung zu psychogenen ruckartigen Innervationsstößen, die in antagonistischen Muskelpaaren stets ein alternierendes (,ballistisches') Innervationsmuster zeigen [21]. Oft folgen Myoklonien und Spasmen unmittelbar aufeinander $(\bullet$ Abb. 3c). Es kommen aber auch rein myoklonische Innervationsmuster vor ( $\bullet$ Abb. 4). Diese können über längere Zeit mit gleich bleibender Frequenz und Amplitude anhalten und muten dann klinisch wie Zitteranfälle an. In vielen Fällen ist die Frequenz der Myoklonien so hoch (10-15/s), dass bei bloßer Betrachtung der Eindruck einer tonischen Kontraktion entsteht - ähnlich wie beim sogenannten orthostatischen Tremor, der die Stand- und Gangstabilität ebenfalls beeinträchtigt durch eine hypersynchrone myoklonische Aktivierung antagonistischer Muskeln mit hoher Frequenz. Diese ist auch hier prima facie nicht erkennbar, aber tastbar, und wird meist erst durch die EMG Ableitung deutlich [22].

\section{Reflex-Polygrafie}

Die Registrierung spontaner Spasmen und Myoklonien gelingt natürlich keineswegs regelmäßig. Insofern ist man hier auf kasuistische Beobachtungen angewiesen. Methodisch einfacher ist die Registrierung und Analyse der reizinduzierten EMGAktivität. So lassen sich durch Stimulation mit unterschiedlichen Modalitäten lang anhaltende Spasmen auslösen ( $\bullet$ Abb. 5). Die Registrierung offenbart dabei eine durch die Stimulation ausgelöste abrupte Innervation aller Muskeln, die langsam und kontinuierlich abklingt. Die Analyse des abrupten Innervationsbeginns belegt eine myoklonische Aktivierung mit reproduzierbar kurzer Latenz ( $\bullet$ Abb. 6 ). Durch Stimulation des Nervus medianus lassen sich beim Gesunden keine Reflexe in der vorderen und hinteren Rumpfmuskulatur auslösen. Bei Patienten mit SMS und seinen Varianten sieht man in diesem Untersuchungspara-

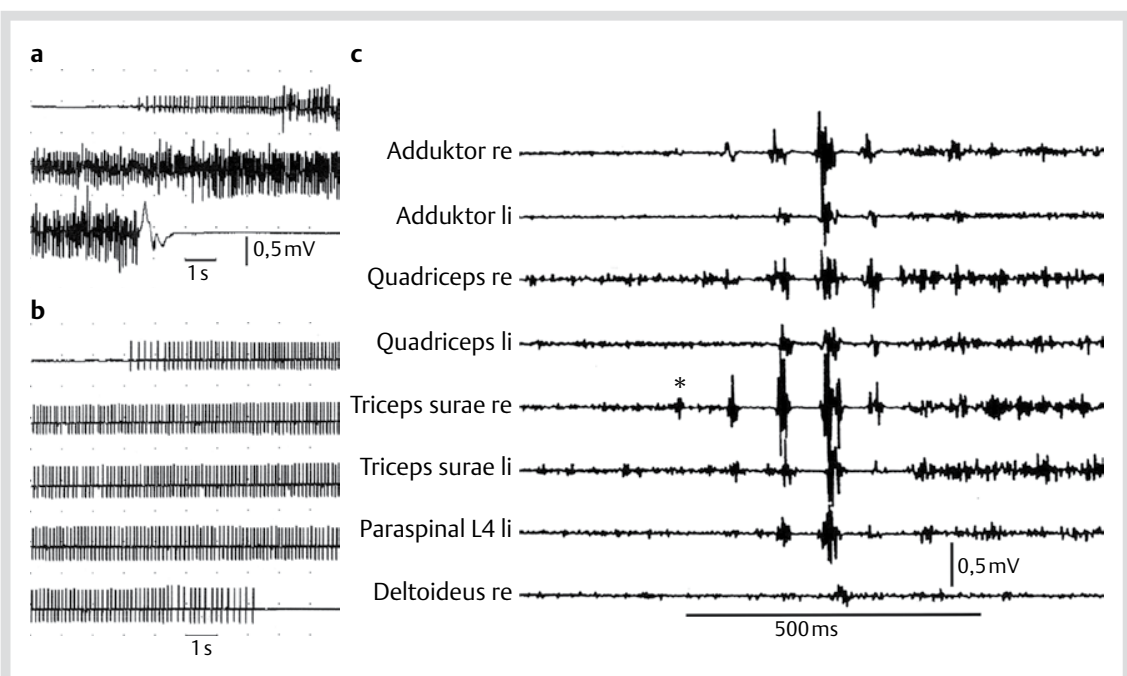

Abb. 3 a, b Spontane Spasmen, abgeleitet mit Nadelelektrode aus dem M. rectus femoris. Patient mit klassischem SMS mit GAD-Antikörpern. c Spontaner Spasmus in der beidseitigen Bein- und Rumpfmuskulatur, eingeleitet durch eine Salve von 5 hypersynchronen Myoklonien. Patient mit paraneoplastischem PERM und unbekanntem Antikörperstatus, abgeleitet im Status spasmodicus. Die Salve beginnt mit einem Burst niedriger Amplitude im M. triceps surae rechts $\left({ }^{*}\right)$. Mit jedem weiteren EMG-Burst wächst die Amplitude an, gleichzeitig breitet sich die myoklonische Aktivität immer weiter in die Nachbarmuskeln aus (re>li). Nach dem 5. hypersynchronen Burst beginnt desynchronisierte EMG Aktivität (Spasmus), die über insgesamt 3,7 s abklingt (nicht dargestellt). Beachte die gegenüber der Paraspinalmuskulatur L4 deutlich verzögerte Aktivierung des M. deltoideus, die auf eine Ausbreitung ,von unten nach oben', mithin auf einen spinalen Generator dieser abnormen Aktivität hinweist. Aus [26] mit freundlicher Genehmigung. 


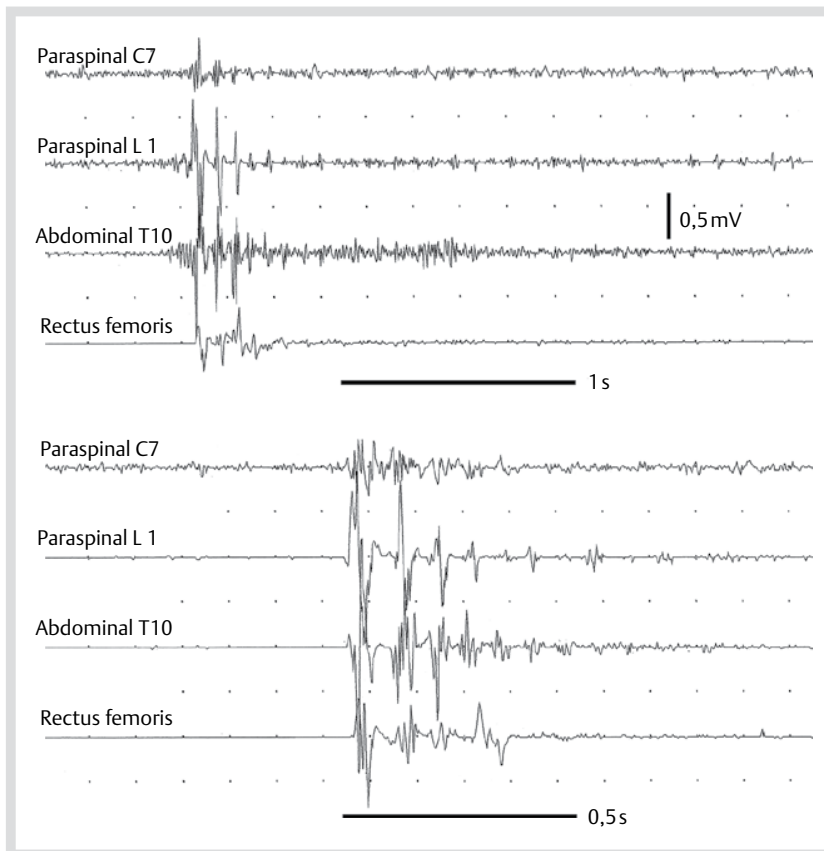

Abb. 42 spontane Salven hochfrequenter Myoklonien ( 12/s), die in der klinischen Beobachtung als myoklonisch eingeleitete Spasmen erscheinen. Patient mit klassischem SMS und GAD-Autoantikörpern. Ableitung mit Oberflächenelektroden. Beachte die in allen Muskeln hypersynchronen Bursts mit minimaler Verzögerung des M. Rectus femoris gegenüber der Paraspinalmuskulatur C7 und L1. Die Salve in der oberen Registrierung hält über das Aufzeichnungsende hinaus an.

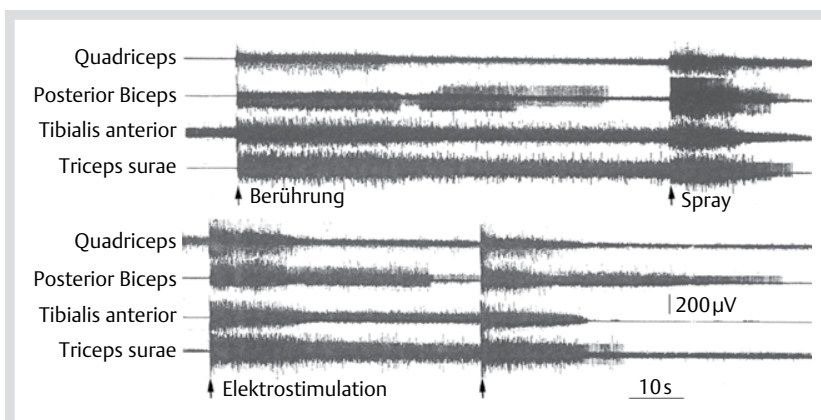

Abb. 5 Fremdreflektorisch ausgelöste Spasmen. Patientin mit PERM und GAD-Autoantikörpern. Kontinuierliche 4-Kanal-EMG- Registrierung mit Nadelelektroden über jeweils knapp 2 min, Reizapplikation (Pfeile) an der gleichseitigen Fußsohle. Aus [6] mit freundlicher Genehmigung.

digma bis zu 5 hypersynchrone Komponenten, die mit kurzer Latenz (60-80 ms) und kurzen Intervallen (um $50 \mathrm{~ms}$ ) aufeinander folgen und dann in allmählich abklingende desynchronisierte (d.h. spasmodische) EMG-Aktivität übergehen. Versucht man die Ausbreitung dieser myoklonisch eingeleiteten Reflexspasmen durch eine longitudinale Anordnung der Ableitelektroden entlang der Körperachse zu bestimmen, so findet man Reflexkomponenten mit kürzester Latenz im thorakolumbalen Übergangsbereich; in der zervikalen und lumbosakralen Paraspinalmuskulatur sind die Latenzen länger ( $\bullet \mathbf{A b b}$. 7). Eine hypothetische Erklärung für diese scheinbar paradoxe Ausbreitung ist, dass der pathologische (disinhibierte?) Generator dieser abnormen Reflexe im unteren Thorakalmark liegt, und von dort aus die Aktivierung der axialen Muskulatur über propriospinale Faserverbindungen nach kaudal und rostral erfolgt. Korrespondie-

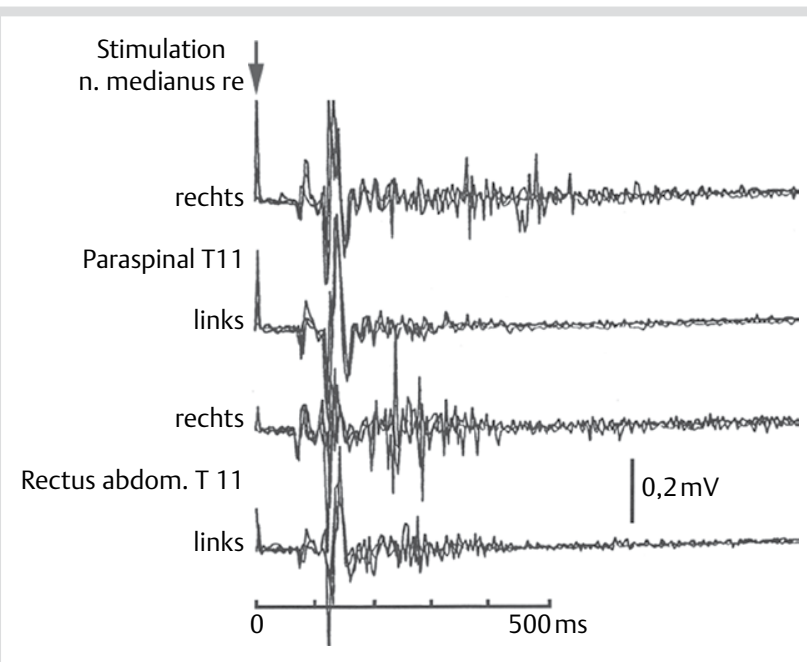

Abb. 6 Myoklonischer Reflex-Spasmus in der vorderen und hinteren Rumpfmuskulatur, ausgelöst durch Stimulation des N. medianus rechts (Reizbeginn: $0 \mathrm{~ms}$ ). Patientin mit klassischem SMS und GAD-Autoantikörpern. Ableitung mit Nadelelektroden. 3 Reizantworten superponiert. Beachte die kurze Latenz ( $70 \mathrm{~ms})$ und die Hypersynchronie der initialen Reflexkomponenten sowie die exakte Reproduzierbarkeit des Reflexmusters. Aus [26] mit freundlicher Genehmigung.

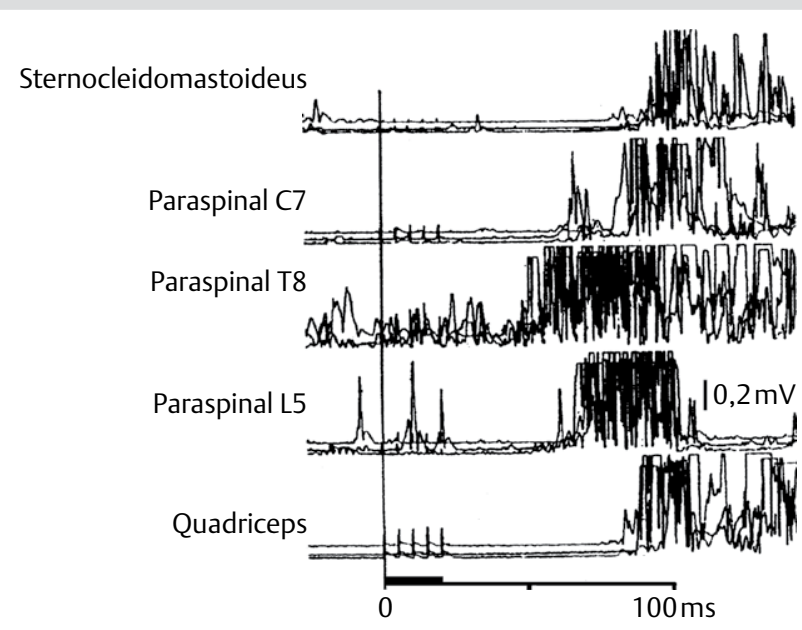

Abb. 7 Myoklonischer Reflex-Spasmus in der rechtsseitigen Rumpf- und rumpfnahen Muskulatur (Ableitung mit Nadelelektroden, EMG gleichgerichtet), ausgelöst durch Elektrostimulation des N. medianus links (Salve aus 5 Impulsen, $200 \mathrm{~Hz}$; Reizdauer markiert auf der Zeitachse). Reizgetriggerte Superposition von 3 Reflexantworten mit kürzester Latenz ( $\sim 50 \mathrm{~ms})$ in der Paraspinalmuskulatur T8. Die nach kaudal und rostral länger werdenden Latenzen sprechen für einen im Segment T8 zu lokalisierenden Fokus, von dem ausgehend sich die abnorme Reflexaktivität nach kaudal und kranial (also bi-direktional) ausbreitet. Aus [26] mit freundlicher Genehmigung.

rende Befunde beobachtet man gelegentlich auch bei der Ableitung spontaner, myoklonisch eingeleiteter Spasmen ( $\mathbf{O A b b}$. 3c).

\section{Kopfretraktionsreflex und Schreckreflex (,Startle')}

Diagnostisch und pathophysiologisch aufschlussreich ist auch die Analyse des Kopfretraktionsreflexes. Dieser atavistische, bei Gesunden nicht auslösbare Reflex besteht klinisch aus einer brüsken Kopfretraktion bei leichtem Beklopfen des Gesichtszentrums, insbesondere des Nasenrückens und der Oberlippe [23]. 


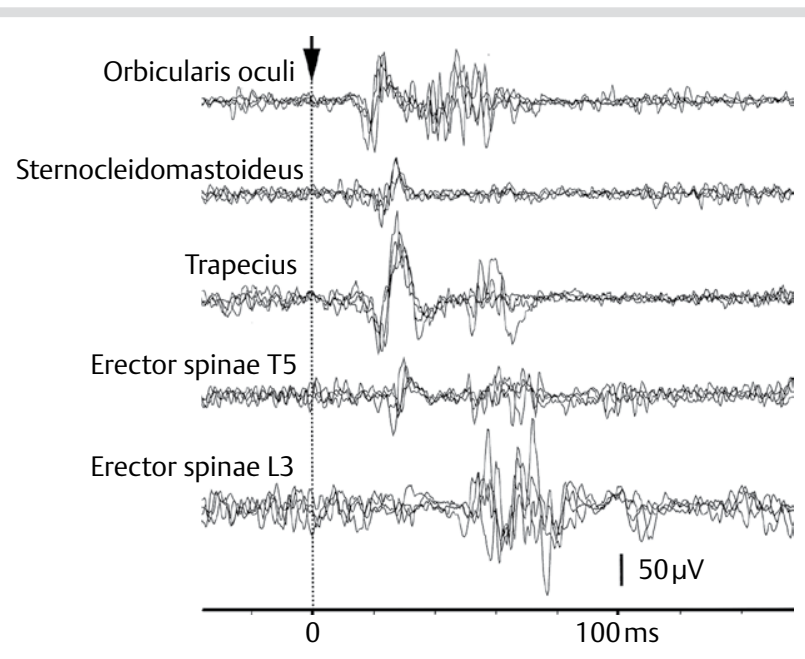

Abb. 8 Elektromyogramm des Kopfretraktionsreflexes bei SMS, ausgelöst durch leichtes Beklopfen des Nasenrückens mit der Fingerkuppe (Pfeilspitze; Frequenz ca. 0,3/s; Kontakttrigger; 0 ms). Simultane Ableitung mit Oberflächenelektroden, 4 Registrierungen superponiert. Das Reflexmuster im M. Orbicularis oculi entspricht dem Blink-Reflex. Das gleichzeitige Auftreten des Reflexes in den Mm. Orbicularis oculi und Trapezius weist darauf hin, dass es sich um eine Reflexirradiation innerhalb des Hirnstamms handelt. Aus [16] mit freundlicher Genehmigung.

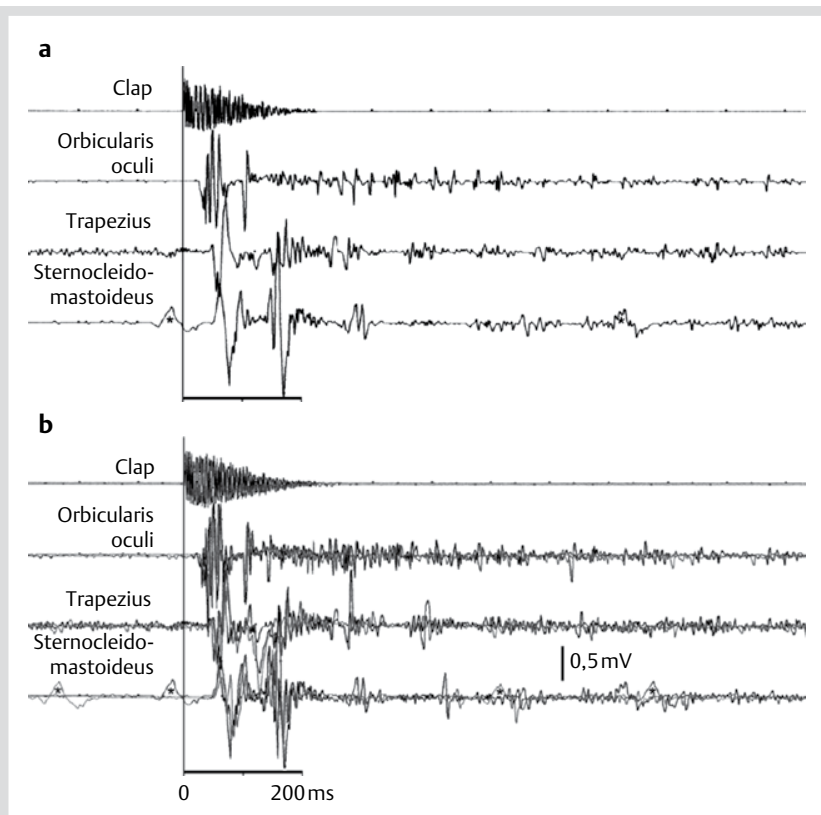

Abb. 9 Pathologisch gesteigerte akustische Schreckreflexe; Patient mit PERM und DPPX-Autoantikörpern. a Simultane Registrierung des akustischen Reizes (Clap) mittels eines am Kragen des Patienten befestigten Mikrofons und des EMG von 3 perikraniellen Muskeln Oberflächenelektroden). ${ }^{*}=$ EKG-Artefakt. $\mathbf{b}$ Reizgetriggerte Überlagerung von 3 konsekutiven Schreckreflexen, ausgelöst mit Intervallen von etwa $2 \mathrm{~s}$. Beachte die kurze Reflexlatenz und die exakte Reproduzierbarkeit der EMG Signale ohne Habituation. Aus [27] mit freundlicher Genehmigung.
In der Heidelberger Kohorte fanden wir diesen Reflex bei knapp 60\% der Patienten mit SMS und seinen Varianten [16] (Infobox 1). Dasselbe Reflexmuster, phänomenologisch und elektromyografisch, wird beim auditiv ausgelösten Erschrecken aktiviert. Anscheinend besteht eine taktile und akustische Konvergenz auf das Reflexzentrum im Hirnstamm. Der Reflex, seine Bahn, seine Modulation durch supraspinale Einflüsse und die Molekularbiologie und Biochemie seiner synaptischen Transmission sind tierexperimentell detailliert untersucht $[24,25]$. Das Reflexzentrum ist der Nucleus caudalis reticularis pontis, der Ursprung des Tractus reticulospinalis, welcher den Hirnstamm monosynaptisch mit den spinalen Motoneuronen verbindet. Die klinisch-neurophysiologische Analyse dieses Reflexes bei Patienten mit SMS und seinen Varianten zeigt, dass er, ähnlich wie der Blinkreflex, aus 2 exzitatorischen Reflexkomponenten mit einer interkalierten Inhibition besteht. Dieses Reflexmuster lässt sich bis in die thorakolumbale Paraspinalmuskulatur verfolgen ( $\bullet$ Abb. 8). Bei taktiler Auslösung ist die Latenz des Reflexes in der Nackenmuskulatur im Mittel nur 2 ms länger als im M. orbicularis oculi. Offensichtlich breitet sich dieser Reflex innerhalb des Hirnstamms aus und irradiiert von dort in die motorischen Kerne der Paraspinalmuskulatur. Ähnlich sind die Befunde bei akustischer Auslösung ( $\boldsymbol{\bullet}$ Abb. $\mathbf{9}$ ) - die Latenzen sind dabei in der Nackenmuskulatur etwas länger als im M. orbicularis oculi. In beiden Fällen ermöglicht die polygrafische EMG Ableitung nicht nur Darstellung und Analyse dieser extrem kurzlatenzigen Fremdreflexe und ihrer Ausbreitung. Sie dokumentiert auch die exakte Reproduzierbarkeit von Reflexlatenz und Reflexmuster als elektrophysiologischen Beleg der organischen Natur dieser Normabweichungen. Bei Patienten mit psychogener Störung lassen sich weder Latenz noch Innervationsmuster reproduzieren ( $\mathbf{O} \mathbf{A b b} . \mathbf{1 0})$.

\section{Kernbotschaft}

$\nabla$

Fluktuierende Rigidität der Rumpfmuskulatur und heftige Spasmen sind das verbindende Merkmal der Stiff Man-Syndrome. Nur bei gut $1 / 3$ der Patienten sind weitere zentralneurologische Störungen zu identifizieren. Ca. 70\% der Patienten haben zusätzliche Autoimmun-Endokrinopathien, und 80-90\% Autoantikörper gegen neuronale Antigene. Die Mehrzahl der routinediagnostischen bildgebenden, elektrophysiologischen und Laboruntersuchungen liefert allenfalls unspezifische Normabweichungen, ermöglicht aber verschiedene Differenzialdiagnosen auszuschließen. Diagnostisch und therapeutisch weiterführend sind das breit angelegte Screening nach neuronalen Autoantikörpern und die klinisch-neurophysiologische Analyse von Spasmen und gesteigerter exterozeptiver Erregbarkeit. Mit der EMG-Polygrafie gelingt vor allem die schwierige Abgrenzung der klinisch oft nicht eindeutig zu klassifizierenden Spasmen und Myoklonien von psychogenen Bewegungsstörungen. Mit elektrophysiologischen Methoden lassen sich darüber hinaus auf mehreren motorischen Regulationsebenen Störungen der neuronalen Inhibition nachweisen, insbesondere im Hirnstamm und Rückenmark. Hierzu korrespondierend wurden als neuronale Antigene vor allem Proteine inhibitorischer Synapsen identifiziert. 


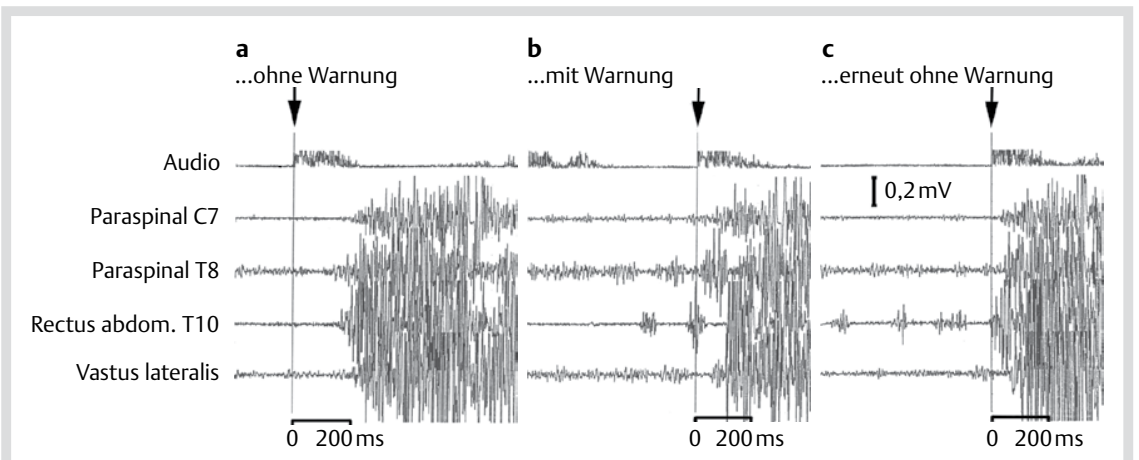

Abb. 10 Akustisch ausgelöster Schreckreflex bei einer Patientin mit psychogener Hyperekplexie. Die Registrierung des Audio-Signals (oben) ist gleichgerichtet, um Überlappungen mit den EMG-Signalen zu vermeiden. Ableitung einseitig mit Oberflächenelektroden von den Paraspinalmuskeln C7 und T8, vom Rectus abdominis T10 und vom Vastus lateralis. Die Patientin liegt mit geschlossenen Augen auf dem Rücken. a Überraschender lauter Knall (Beginn markiert mit vertikaler Linie). b Lauter Knall mit Vorwarnung (Ende der Vorwarnung zu Beginn der Registrierung im Audio-Kanal erkennbar). Beachte die rhythmisch alternierende Vorinnervation in der Rumpfmuskulatur (,Schwung holen'). c Erneuter lauter Knall ohne Vorwarnung. Die rhythmische Vorinnervation in der Abdominalmuskulatur und die unrealistisch kurze Latenz ( $0 \mathrm{~ms}$ !) legen nahe, dass die Patientin mit unvollständig geschlossenen Lidern den Untersucher bei der Auslösung des akustischen Signals beobachtet hat. Aus [28] mit freundlicher Genehmigung.

Interessenkonflikt: H.-M. Meinck erhielt Beraterhonorare von Mallinckrodt LLC, Hazelwood, MO 63042, USA und Vortragshonorare von GlaxoSmithKline, München und Euroimmun, Lübeck.

\section{Literatur}

1 Moersch HF, Woltman HW. Progressive fluctuating muscular rigidity and spasm ("stiff-man" syndrome); report of a case and some observations in 13 other cases. Proc Staff Meet Mayo Clin 1956; 31: 421-427

2 Meinck HM, Ricker K, Hülser PJ et al. Stiff man syndrome: clinical and laboratory findings in eight patients. J Neurol 1994; 241: 157-166

3 Barker RA, Revesz T, Thom $M$ et al. Review of 23 patients affected by the stiff man syndrome: clinical subdivision into stiff trunk (man) syndrome, stiff limb syndrome, and progressive encephalomyelitis with rigidity. J Neurol Neurosurg Psychiatry 1998; 65: 633-640

4 Meinck HM, Thompson PD. Stiff man syndrome and related conditions. Mov Disord 2002; 17: 853-866

5 Espay AJ, Chen R. Rigidity and spasms from autoimmune encephalomyelopathies: stiff-person syndrome. Muscle Nerve 2006; 34: 677-690

6 Meinck HM. Das Stiff man Syndrom und seine Varianten. Nervenarzt 2013; 84: 450-454

7 Campbell AM, Garland H. Subacute myoclonic spinal neuronitis. J Neurol Neurosurg Psychiatry. 1956; 19: 268-274

8 Whiteley AM, Swash M, Urich $H$. Progressive encephalomyelitis with rigidity. Brain 1976; 99: 27-42

9 Meinck HM. Stiff man syndrome. CNS Drugs 2001; 15: 515-526

10 Seissler J, Scherbaum WA. Stiff-man syndrom aus internistischer und immunologischer Sicht. Internist 2000; 41: 460-466

11 Geis $C$, Weishaupt A, Hallermann $S$ et al. Stiff person syndrome-associated autoantibodies to amphiphysin mediate reduced GABAergic inhibition. Brain 2010; 133: 3166-3180

12 Hansen N, Grünewald B, Weishaupt A et al. Human Stiff person syndrome IgG-containing high-titer anti-GAD65 autoantibodies induce motor dysfunction in rats. Exp Neurol 2013; 239: 202-209

13 Alexopoulos H, Dalakas MC. Immunology of stiff person syndrome and other GAD-associated neurological disorders. Expert Rev Clin Immunol 2013; 9: 1043-1053
14 Saiz A, Blanco Y, Sabater L et al. Spectrum of neurological syndromes associated with glutamic acid decarboxylase antibodies: diagnostic clues for this association. Brain 2008; 131: 2553-2563

15 McKeon A, Martinez-Hernandez E, Lancaster E et al. Glycine receptor autoimmune spectrum with stiff-man syndrome phenotype. JAMA Neurol 2013; 70: 44-50

16 Berger C, Meinck HM. Head retraction reflex in stiff-man syndrome and related disorders. Mov Disord 2003; 18: 906-911

17 Khasani S, Becker K, Meinck HM. Hyperekplexia and stiff-man syndrome: abnormal brainstem reflexes suggest a physiological relationship. J Neurol Neurosurg Psychiatry 2004; 75: 1265-1269

18 Schulte-Mattler WJ, Zierz S. Stiff-man syndrome: abnormal late responses upon transcranial magnetic stimulation. Muscle Nerve 1996; 19: 926-927

19 Koerner $C$, Wieland B, Richter $W$ et al. Stiff-person syndromes: motor cortex hyperexcitability correlates with anti-GAD autoimmunity. Neurology 2004; 62: 1357-1362

20 Floeter MK, Valls-Solé J, Toro $C$ et al. Physiologic studies of spinal inhibitory circuits in patients with stiff-person syndrome. Neurology 1998; 51: 85-93

21 Brown $P$, Thompson PD. Electrophysiological aids to the diagnosis of psychogenic jerks, spasms, and tremor. Mov Disord 2001; 16: 595-599

22 Muthuraman M, Hellriegel $H$, Paschen $S$ et al. The central oscillatory network of orthostatic tremor. Mov Disord 2013; 28: 1424-1430

23 Wartenberg $R$. Head retraction reflex. Calif Med 1949; 70: 382

24 Koch $M$. The neurobiology of startle. Prog Neurobiol 1999; 59: 107-128

25 Davies JS, Chung SK, Thomas RH et al. The glycinergic system in human startle disease: a genetic screening approach. Front Mol Neurosci doi:10.3389/fnmol2010.00008

26 Meinck HM, Ricker K, Hülser PJ et al. Stiff man syndrome: neurophysiological findings in eight patients. J Neurol 1995; 242: 134-142

27 Balint B, Jarius S, Nagel S et al. Progressive encephalomyelitis with rigidity and myoclonus: a new variant with DPPX antibodies. Neurology 2014; 82: 1521-1528

28 Meinck HM. Krämpfe, Spasmen und verwandte Symptome. In: Schmitz B, Tettenborn B, Hrsg. Paroxysmale Störungen in der Neurologie Heidelberg: Springer 2004; 176-193 